\title{
Strangeness at Intermediate Baryon Density
}

\author{
David Tlusty ${ }^{1, \star}$ \\ ${ }^{1}$ Physics \& Astronomy Department, Rice University, Houston TX, USA
}

\begin{abstract}
.
Exploration of the QCD phase diagram has been one of the main programs of contemporary nuclear physics. The intermediate baryon density region covers a broad range of the baryon chemical potential, between 100 and $700 \mathrm{MeV}$, and is expected to include a possible critial point at the end of a phase equilibrium curve between the hadron gas and quark gluon plasma phases. Experimental programs at the SPS and RHIC facilities have provided valuable insights in this range. These proceedings motivate the exploration of the QCD phase diagram through the use of strangeness. A selection of relevant experimental results from RHIC and SPS beam energy scan programs with associated theoretical predictions is presented along with a discussion of possible physical conclusions and future plans.
\end{abstract}

\section{Introduction}

Finite temperature lattice Quantum Chromodynamics (QCD) calculations predict [1] a cross-over from a hadronic to a QGP phase at a vanishing baryon chemical potential $\mu_{B}$ and temperature $T=$ $154 \pm 9 \mathrm{MeV}$ [2]. Several QCD-based calculations [3, 4] show that at lower $T$ and higher $\mu_{B}$ a first-order phase transition may take place. The point in the QCD phase diagram, where the first order phase transition ends, is the QCD critical point [5]. In the low- $T$, and low and intermediate $\mu_{B}$ phase, interacting hadronic matter in the ground state can be well described in terms of a gas of non-interacting hadrons and resonances - the Hadron Resonance Gas (HRG) model [6].

The availability of more precise experimental data now allows to better discriminate between models. The lattice QCD prediction power is still very limited at non-zero $\mu_{B}$, hence, experimental exploration becomes especially vital. These proceedings will focus on discussion of the intermediate $\mu_{B}$ region, which covers a wide $\mu_{B}$ range, roughly spanning between $100 \mathrm{MeV}$ and $700 \mathrm{MeV}$. The intermediate baryon density $\mu_{B}$ region is accessible through experiments at Relativistic Heavy Ion Collider (RHIC), Super Proton Synchrotron (SPS), and in the future Facility for Antiproton and Ion Research (FAIR) which will be able to access also high $\mu_{B}$ region. Both SPS and RHIC have been running centre-of-mass energy $\sqrt{s_{N N}}$ and system size scans to fully explore the region.

One of the most important experimental tools to probe the QCD phase diagram is the study of strange particle production. Strange quarks are strongly interacting particles from the second quark flavor generation. In a QGP, strangeness can be easily produced as strange-antistrange quark pairs via gluon-gluon or quark-antiquark fusion [7], where the minimum momentum transfer $Q$ for this

\footnotetext{
${ }^{\star}$ e-mail: tlusty@ rice.edu
} 
process is $Q \approx 200 \mathrm{MeV}$, while the lowest $Q$ process in a hadron gas, $n+n \longrightarrow n+\Lambda+K$, needs $670 \mathrm{MeV}$. Strangeness is also abundant since nearly $20 \%$ of all energy content of QGP is transferred to the production of strangeness when chemical equilibrium is reached [8].

Studies of strange hadron production in the energy and system size scans help to understand many crucial questions; to name a few: what is the temperature and density of strongly interacting matter formed in heavy ion collisions, what types of phase transitions occur, is there a critical point in the QCD phase diagram, and at which collision energy do the QGP signals turn off.

The next sections will present a selection of experimental results and theoretical calculations relevant to the discussion.

\section{Selection of Experimental Results and Theoretical Calculations}

\subsection{Flavor Hierarchy in the Deconfinement Transition of QCD}
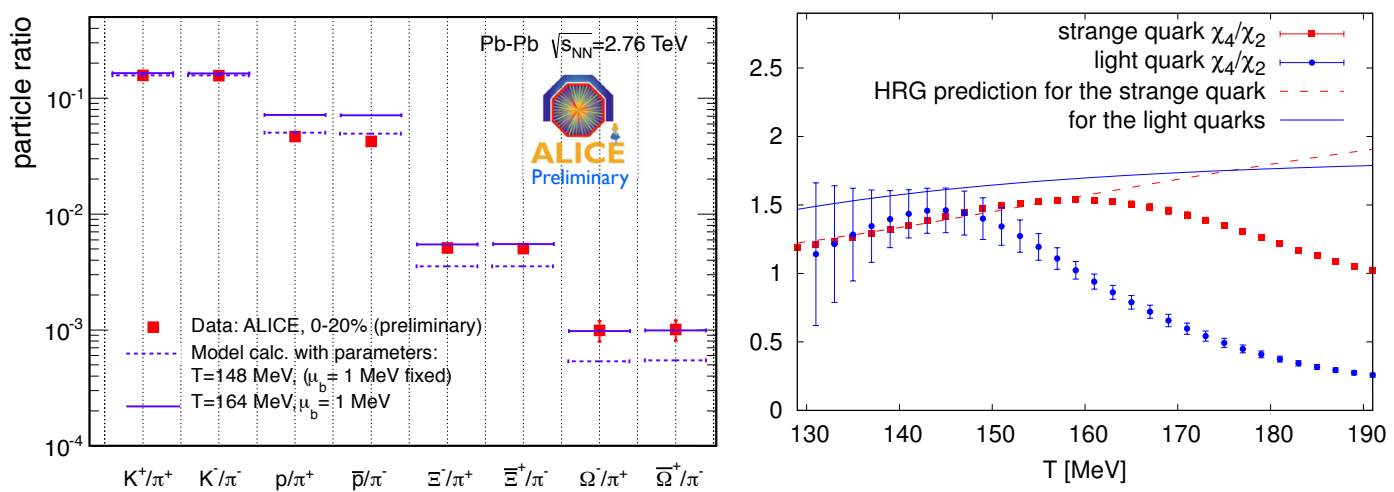

Figure 1. Left panel: Hadron-production ratios from $\mathrm{Pb}+\mathrm{Pb}$ collisions at $\sqrt{s_{N N}}=2.76 \mathrm{TeV}$ compared to the statistical hadronization model predictions with parameters $T=148,164 \mathrm{MeV}$ and $\mu_{B}=1 \mathrm{MeV}$. Right Panel: The $T$ dependence of the susceptibility ratios $\chi_{2} / \chi_{4}$ for light and strange quarks in the continuum limit. The lattice data are compared to HRG calculations. Left panel taken from Ref [9] and right panel from Ref [15].

At the Strangeness in Quark Matter conference 2011, the ALICE Collaboration has presented results of particle production ratios [9]: $K^{+} / \pi^{+}, K^{-} / \pi^{-}, p / \pi^{+}, \bar{p} / \pi^{-}, \Xi^{-} / \pi^{+}, \bar{\Xi}^{+} / \pi^{-}, \Omega^{-} / \pi^{+}$, and $\bar{\Omega}^{+} / \pi^{-}$, (i.e., ratios of strange-to-light and light-to-light hadrons) together with their thermal statistical model predictions $[10,11]$ at chemical freeze-out temperatures of $T_{\mathrm{ch}}=164$ and $148 \mathrm{MeV}$. As shown in Fig. 1 (left panel), the thermal model prediction with $T_{\mathrm{ch}}=164 \mathrm{MeV}$ describes the strange-to-light ratios while the prediction with $T_{\mathrm{ch}}=148 \mathrm{MeV}$ describes $p / \pi^{+}, \bar{p} / \pi^{-}, K^{+} / \pi^{+}$, and $K^{-} / \pi^{-}$. This is indicative of a separation of chemical freeze-out temperatures between light and strange quark hadrons. Since the ALICE results came from $\mathrm{Pb}+\mathrm{Pb}$ collisions at $\sqrt{s_{N N}}=2.7 \mathrm{TeV}$, the $\mu_{B}$ was nearly 0 and the results are suited for comparisons with lattice QCD calculations of higher order quark number susceptibilities $\chi_{n}$ [12-14] for light and strange quarks [15]. Figure 1 (right panel) shows the dependency of the susceptibility ratios $\chi_{4} / \chi_{2}$ for light and strange quarks on temperature $T$ together with corresponding HRG model predictions. The critical temperature $T_{c}$ is assumed to be the temperature where the lattice QCD calculated $\chi_{4} / \chi_{2}$ and HRG prediction begin to differ. The choice of susceptibility ratio has two advantages. The susceptibility ratio cancels finite volume effects and is proportional to some 
experimental observables which will be discussed later. As one can observe from from Fig. 1 (right panel), the $T_{c} \approx 160 \mathrm{MeV}$ for strange quarks differ from the $T_{c} \approx 145 \mathrm{MeV}$ for the light quarks and both are very close to the corresponding $T_{\text {ch }}$ from the thermal statistical model predictions for the ALICE data. This suggests a flavor hierarchy in the deconfinement transition of QCD.

The previous paragraph introduces a flavor hierarchy in the deconfinement transition of QCD at low $\mu_{B}$. At intermediate $\mu_{B}$ a pattern has been observed similar to ALICE's observations at low $\mu_{B}$. The STAR Collaboration recently published [16] its results on bulk properties from the RHIC BES. Figure 2 shows the comparison of extracted freeze-out parameters in Au+Au collisions at $\sqrt{s_{N N}}=39$ $\mathrm{GeV}$ for a Grand Canonical Ensemble (GCE) using particle yields as input to the THERMUS model [17]. Results were compared for four different sets, specified in the legend of Fig. 2, of particle yields used as input for fitting. When only $\pi, K$, and $p$ yields are used in the fit, the temperature obtained is lower compared to other sets that include strange hadron yields.

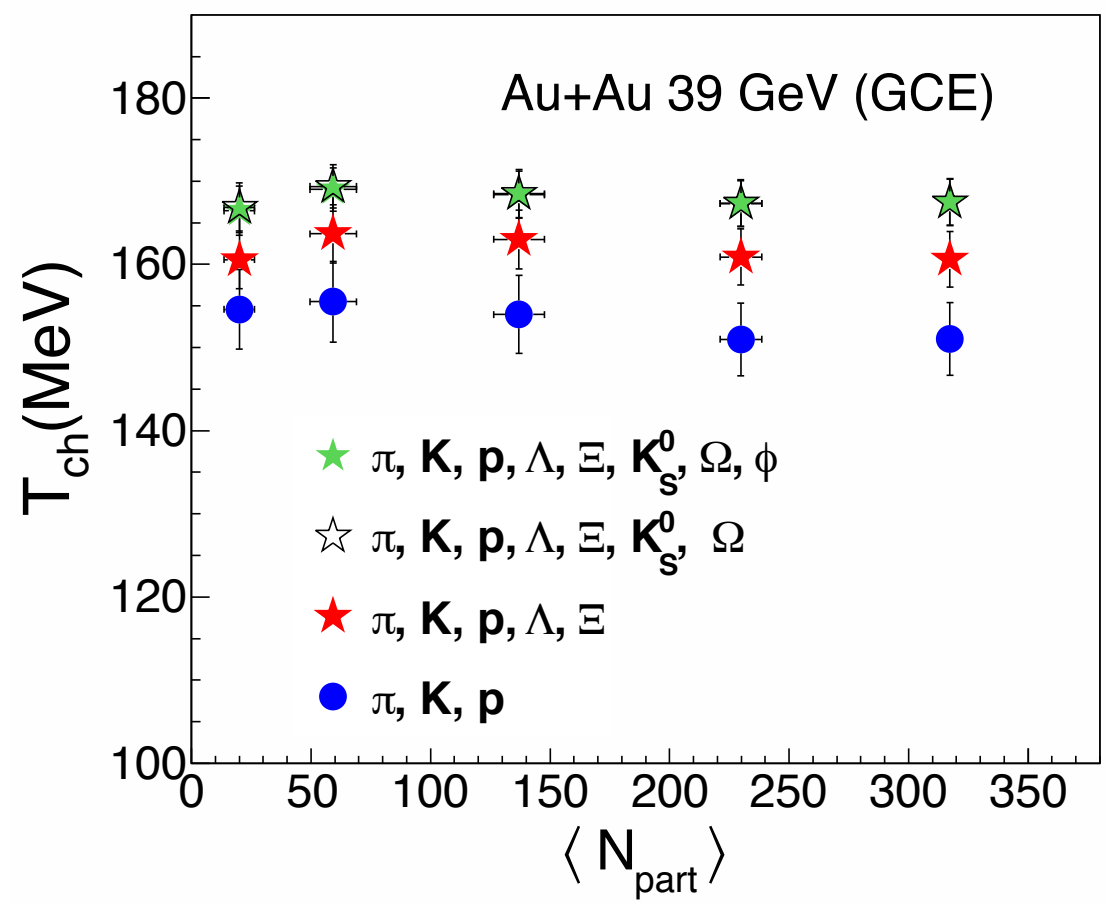

Figure 2. Extracted chemical freeze-out temperature for GCE using particle yields as input for THERMUS model. Results are compared for Au+Au collisions at $\sqrt{s_{N N}}=39 \mathrm{GeV}$ for four different sets of particle yields used in fitting. Figure taken from Ref [16].

\subsection{Cumulant Ratios of Net-Particle Multiplicity}

Fluctuations of conserved quantities are sensitive observables in the study of phase transitions in the QCD matter and critical point [18]. UrQMD model [19] based studies of centrality and energy dependence of various order cumulants and cumulant ratios (up to fourth order) of net-proton, netcharge, and net-kaon multiplicity distributions in $\mathrm{Au}+\mathrm{Au}$ collisions at $\sqrt{s_{N N}}=7.7,11.5,19.6,27$, 
$39,62.4$, and $200 \mathrm{GeV}$ were presented in [18]. These calculations were compared with the data from RHIC Beam Energy Scan (BES) published in [20, 21].

A distribution function can be characterized by the various moments, such as mean $M$, variance $\sigma^{2}$, skewness $S$, and kurtosis $\kappa$. The moments products $\kappa \sigma^{2}$ and $S \sigma$ are directly related to the ratios of various order susceptibilities as

$$
\kappa \sigma^{2}=\frac{\chi_{4}}{\chi_{2}} \quad S \sigma=\frac{\chi_{3}}{\chi_{2}}
$$

As mentioned in Sect. 2.1, using the ratios allows finite volume effects to nearly cancel out and provides for a direct comparison with lattice QCD predictions.
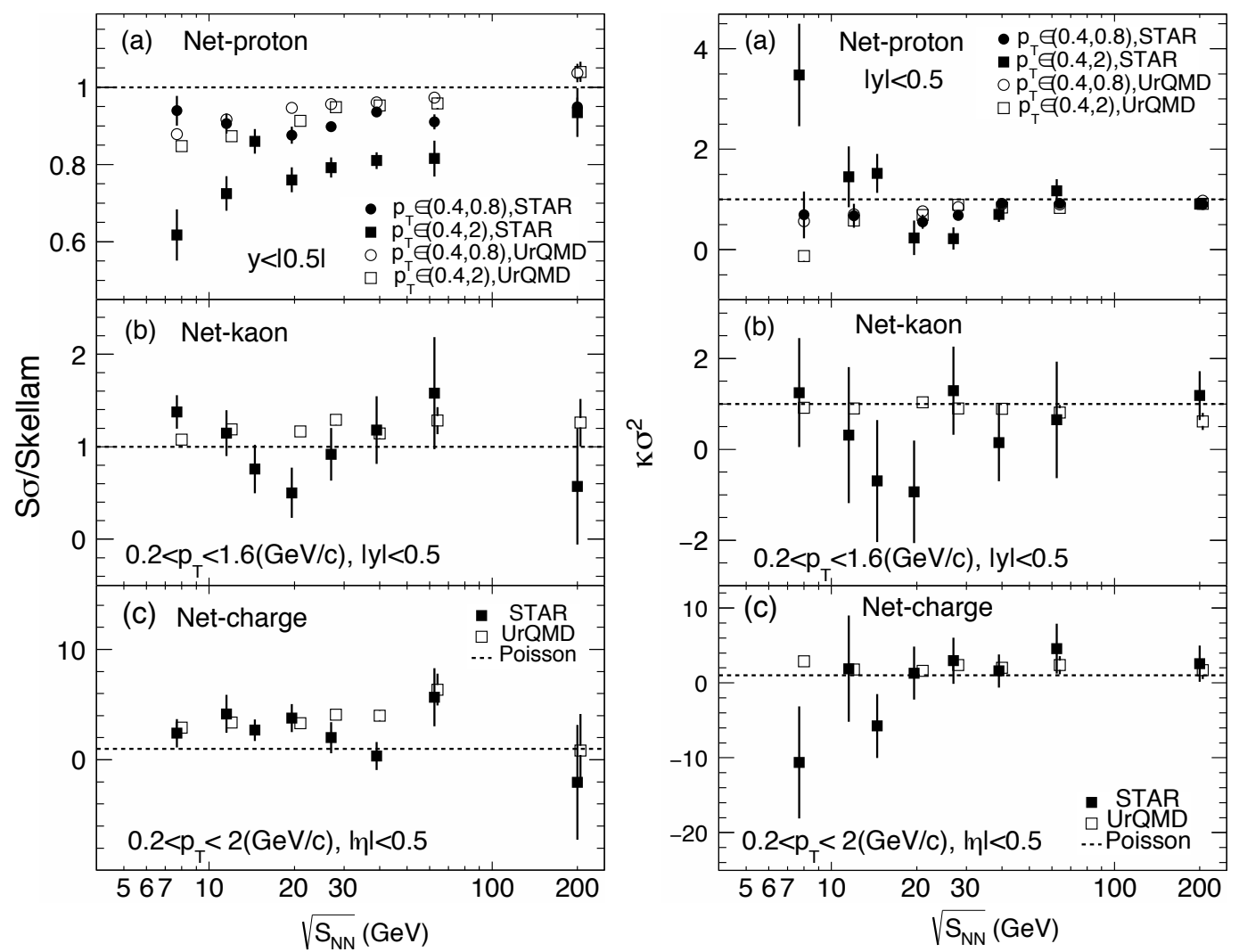

Figure 3. Energy dependence of cumulant ratios $S \sigma$ (left panel), $\kappa \sigma^{2}$ (right panel) of net-proton, net-charge, and net-kaon multiplicity distributions for the $\mathrm{Au}+\mathrm{Au}$ collision at $\sqrt{s_{N N}}=7.7$ to $200 \mathrm{GeV}$. The solid markers represent the results from the STAR measurement, the open markers represent results from the UrQMD calculation. The dashed lines denote the Poisson expectations for the STAR data. Figure taken from Ref [18].

Figure 3 shows the energy dependence of cumulant ratios $S \sigma, \kappa \sigma^{2}$ of net-proton, net-charge, and net-kaon multiplicity distributions of the 5\% most central $\mathrm{Au}+\mathrm{Au}$ collisions in RHIC BES energies from the STAR experiment [20,21] and UrQMD calculations [18]. The non-monotonic energy dependence of the net-proton $\kappa \sigma^{2}$ and net-kaon $S \sigma$ contrasts with the monotonic behavior of UrQMD 
predictions. This discrepancy might suggest critical behavior since the critical physics was not implemented in the UrQMD. It is important to reduce uncertainties of these measurements, which is expected in RHIC BES phase II [22], to get to more conclusive results.

\subsection{Study of the Onset of Deconfinement}

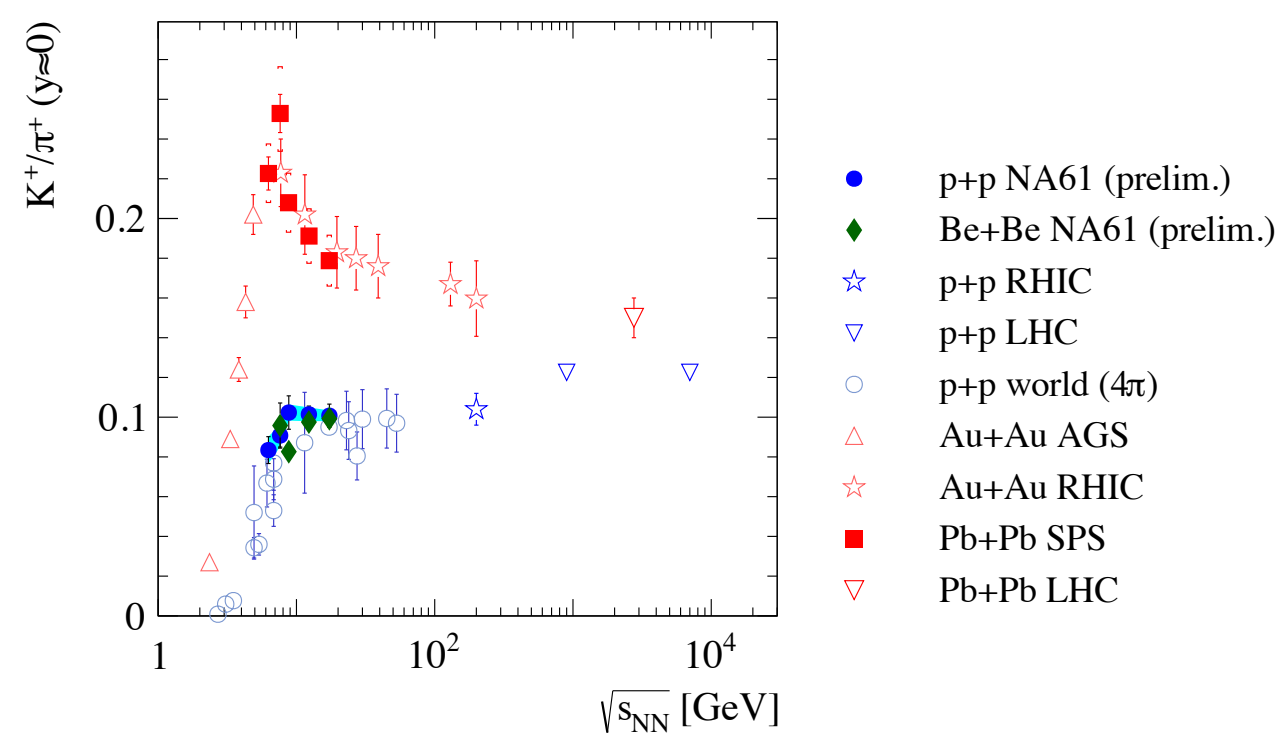

Figure 4. Energy dependence of the positively charged kaon multiplicity divided by corresponding charged pion multiplicity at mid-rapidity. Figure taken from Ref [23]

At the SPS, measurements of hadron production in a two-dimensional scan in beam momentum (13-158 AGeV/c) and system size $\left(\mathrm{p}+\mathrm{p}, \mathrm{p}+\mathrm{Pb},{ }^{7} \mathrm{Be}+{ }^{9} \mathrm{Be}, \mathrm{Ar}+\mathrm{Sc}, \mathrm{Xe}+\mathrm{La}\right.$ and $\mathrm{Pb}+\mathrm{Pb}$ ) were conducted. New results from $\mathrm{p}+\mathrm{p}$ and ${ }^{7} \mathrm{Be}+{ }^{9} \mathrm{Be}$ were shown at Quark Matter 2017 conference [23], presenting the energy dependence of the $K^{+} / \pi^{+}$multiplicity ratio at mid-rapidity. As shown in Fig. 4 , the data from ${ }^{7} \mathrm{Be}+{ }^{9} \mathrm{Be}$ do not show a "horn" structure as data from heavier collision system do (average number of participants in ${ }^{7} \mathrm{Be}+{ }^{9} \mathrm{Be}$ collisions is around 10). This observation constrains size of system to study location of onset of deconfinement energy. The energy dependence of the inverse slope parameter of the transverse mass distribution of charged kaons, as shown in Fig. 5, shows the step structure in both $\mathrm{p}+\mathrm{p}$ and ${ }^{7} \mathrm{Be}+{ }^{9} \mathrm{Be}$. The step structure was predicted by the Statistical Model of the Early Stage (SMES) [24] and 3+1 Hydro model [25] as a signature of the onset of deconfinement.

The UrQMD based transport approach [26] provides further insights. It concludes that the "step" is only reproduced if a first order phase transition with a large latent heat is applied or the equation of state is effectively softened due to non-equilibrium effects in the hadronic transport calculation.

\subsection{Ratios of The Freeze-out Parameters}

The experimental results of ratios of the freeze-out parameters $\mu_{S} / \mu_{B}$ and $\mu_{B} / T$ ( $\mu_{S}$ is strange chemical potential) from the NA57 experiment [27] and the STAR experiment [28] can be reproduced by 

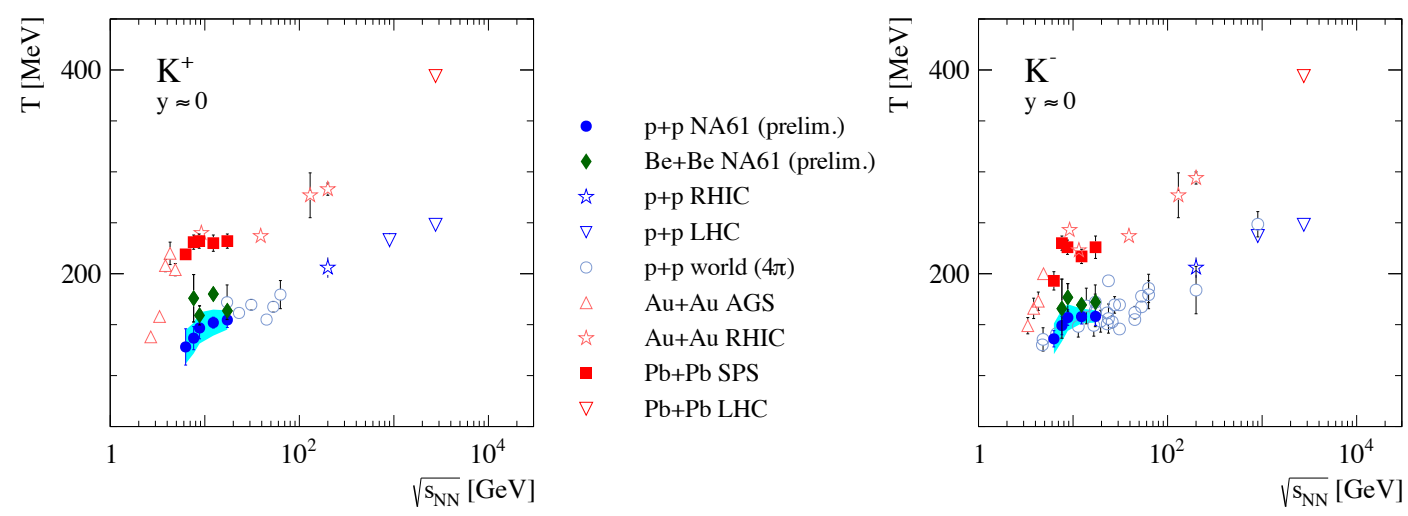

Figure 5. Energy dependence of the inverse slope parameter of the transverse mass distribution at mid-rapidity for charged kaons. The NA61/SHINE results on $\mathrm{p}+\mathrm{p}$ interactions (full blue circles) and new results on $\mathrm{Be}+\mathrm{Be}$ (full green diamonds) collisions are compared with world data on $\mathrm{p}+\mathrm{p}$ and heavy ion $(\mathrm{Pb}+\mathrm{Pb}$ and $\mathrm{Au}+\mathrm{Au})$ reactions. Figure taken from Ref [23]

a HRG model, as discussed in [29]. The HRG model prediction is in better agreement with lattice QCD calculations [29] if additional (strange) hadron resonances are included which are predicted by the quark model $[30,31]$ but until today unobserved (such a setup of the HRG model is denoted as QM-HRG). The $\mu_{S} / \mu_{B}$ and $\mu_{B} / T$ ratios were obtained by a statistical thermal model [32] fitting data for strange-baryon ratios $\bar{\Lambda} / \Lambda, \Xi^{+} / \Xi^{-}$, and $\Omega^{+} / \Omega^{-}$and are shown in Fig. 6 together with lattice QCD predictions, and HRG predictions. One HRG predictions assumes only experimentally discovered hadrons (PDG-HRG) and another is the QM-HRG. As Fig. 6 presents, the QM-HRG predictions match the experimental data and the lattice QCD at the temperatures consistent with the lattice QCD and the data while the PDG-HRG matches at temperatures significantly higher. Hence, it appears that additional, experimentally yet-to-be observed strange hadrons become thermodynamically relevant in the vicinity of the QCD crossover.

\section{Summary and Future Plans}

Recent results shown at this conference have underlined once again the relevance of the study of strangeness at intermediate $\mu_{B}$. There are indications of flavor hierarchy with respect to the chemical freeze-out [33]. Hadrons containing strange quarks appear to hadronize earlier than the hadrons containing only quarks from the first generation. This has been predicted by lattice QCD at $\mu_{B}=0$ and experimentally observed by the ALICE Collaboration [9] as well as at the intermediate $\mu_{B}$ by the STAR Collaboration [16].

In order to describe the freeze-out parameter ratios consistently with the experimental results from STAR and NA57, a Hadron Resonance Gas needs to assume additional, yet-to-be observed strange hadrons. The experimental results are also predicted by lattice QCD.

The NA61 Collaboration has shown intriguing results from ${ }^{7} \mathrm{Be}+{ }^{9} \mathrm{Be}$ collisions. A comparison with its results on $K^{+} / \pi^{+}$ratios shows a remarkably similar structure, which departs from the "horn".

The RHIC BES Phase II is scheduled in years 2019 and 2020. It will provide substantially higher statistics thanks to the accelerator upgrades [22]. Detector upgrades will further reduce systematic uncertainties of measurements. The fixed target program at STAR will allow for the QCD phase 


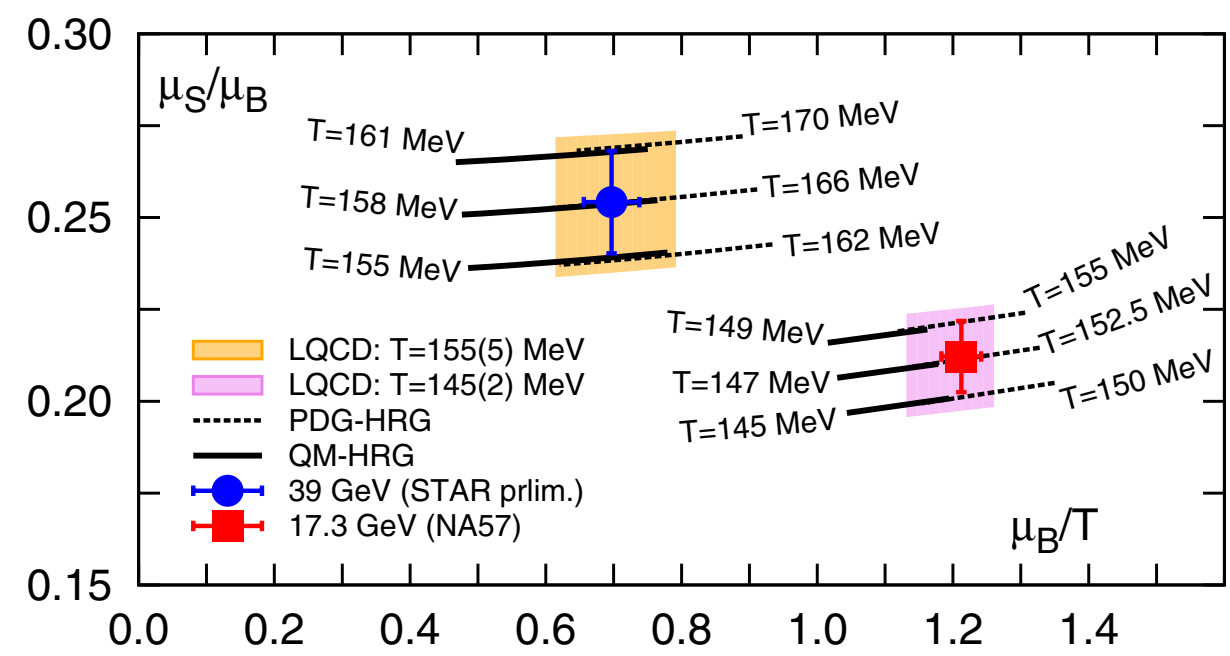

Figure 6. Values of $\mu_{S} / \mu_{B}$ and $\mu_{B} / T$ extracted from fits to multiple strange hadrons yields (blue circle at $\sqrt{s_{N N}}=$ $39 \mathrm{GeV}$, red square at $\sqrt{s_{N N}}=17.3 \mathrm{GeV}$ ) are compared to the lattice QCD calculations (shaded bands) as well as to the QM-HRG (solid lines) and PDG-HRG (dotted lines) models. Figure taken from Ref [29].

space diagram exploration to be extended to $\mu_{B} \simeq 720 \mathrm{MeV}$. At the SPS, NA61 will upgrade its data acquisition system to allow for a $1 \mathrm{kHz}$ readout. This is expected to be ready in 2020 and will allow a high statistics beam momentum scan with $\mathrm{Pb}+\mathrm{Pb}$ collisions for precise measurement of multi-strange hyperon production. All these upgrades will help further constrain the search for a critical point, and the type of phase transition in the intermediated baryon density region.

Acknowledgements This work is part of and supported by U.S. Department of Energy under contract number DE-FG02-10ER41666.

\section{References}

[1] Y. Aoki et al., Nature 443, 675 (2006)

[2] A. Bazavov et al., Phys. Rev. D 80, 054503 (2012)

[3] S. Ejiri, Phys. Rev. D 78, 074507 (2008)

[4] E. S. Bowman and J. I. Kapusta, Phys. Rev. C 79, 015202 (2009)

[5] S. Gupta et al., Science 332, 1525 (2011)

[6] P. Braun-Munzinger, K. Redlich, and J. Stachel, Quark-Gluon Plasma, edited by R. C. Hwa and X.-N. Wang (World Scientific, Singapore, 2004), Vol. 3, p. 491.

[7] J. Rafelski, B. Müller, Phys. Rev. Lett. 48, 1066 (1982)

[8] J. Rafelski, Acta Physica Polonica B 43, 829 (2012)

[9] R. Preghenella (ALICE Collaboration), Acta Physica Polonica B 43, 555 (2012)

[10] J. Cleymans et al., Phys. Rev. C 74, 034903 (2006)

[11] A. Andronic et al., Phys. Lett. B 673, 142 (2009)

[12] S. Gottlieb et al., Phys. Rev. Lett 59, 2247 (1987)

[13] R. V. Gavai and S. Gupta, Phys. Rev. D 68, 034506 (2003) 
[14] S. Datta et al., Phys. Rev. D 95, 054512 (2017)

[15] R. Bellwied et al., Phys. Rev. Lett. 111, 202302 (2013)

[16] L. Adamczyk et al. (STAR Collaboration), Phys. Rev. C 96, 044904 (2017)

[17] S. Wheaton et al., Comput. Phys. Commun. 180, 84 (2009)

[18] J. Xu et al., Phys. Rev. C 94, 024901 (2016)

[19] M. Bleicher et al., J. Phys. G: Nucl. Part. Phys. 25, 1859 (1999)

[20] L. Adamczyk et al. (STAR Collaboration), Phys. Rev. Lett. 113, 092301 (2014)

[21] L. Adamczyk et al. (STAR Collaboration), Phys. Rev. Lett. 112, 032302 (2014)

[22] STAR Collaboration, Studying the Phase Diagram of QCD Matter at RHIC, Star Note 0598 (2014)

[23] A. Aduszkiewicz (NA61/SHINE Collaboration), Nucl. Phys. A 967, 35 (2017)

[24] M. Gazdzicki and M. I. Gorenstein, Acta Phys. Polon. B 30, 2705 (1999)

[25] M. Gazdzicki et al., Braz. Jour. Phys. 34, 322 (2004)

[26] H. Petersen et al., J. Phys. G 36, 055104 (2009)

[27] F. Antinori et al. (NA57 Collaboration), Phys. Lett. B 595, 68 (2004)

[28] F. Zhao (STAR Collaboration), Proc. Sci., CPOD2013 (2013) 036

[29] A. Bazavov et al., Phys. Rev. Lett. 113, 072001 (2014)

[30] S. Capstick and N. Isgur, Phys. Rev. D 34, 2809 (1986)

[31] D. Ebert et al., Phys. Rev. D 79, 114029 (2009)

[32] J. Cleymans, arXiv:nucl-th/9704046v1

[33] R. Belwied, Sequential Strangeness Freeze-out, SQM2017 proceedings 